\title{
LOS PARADIGMAS \\ EMERGENTES EN EL DISEÑO \\ DE LA ARQUITECTURA \\ POS-COVID-I 9 EN \\ CiUdAd JUÁREZ: ESPACIOS \\ SINCRÓNICOS Y ASINCRÓNICOS
}

\author{
The Emerging Paradigms in Post Covid-i9 \\ Architectural Design in Ciudad Juárez: \\ Synchronous and AsynChronous SPACES
}

LEÓN FELIPE DURÓN MORALES ${ }^{1}$ Y LETICIA PEÑA BARRERA ${ }^{2}$

\section{RESUMEN}

1 l confinamiento originado para mitigar la pandemia de la COVID-19 tiene como repercusión efectos en distintas actividades de la vida cotidiana. En esta investigación se busca identificar transformaciones generadas en los hogares de Ciudad Juárez, Chihuahua, a través de las modificaciones en uso y espacio de áreas esenciales. La metodología utilizada es de tipo mixto, aplicando herramientas cualitativas y cuantitativas, que dan cuenta del empleo de espacios y sus transformaciones durante el confinamiento por la COVID-19. Los resultados observados son cambios identificados que generan espacios asincrónicos en 
el ámbito cotidiano de la familia, modificando la organización y estructura previa del uso de la vivienda.

Palabras clave: sincrónico; asincrónico; espacio doméstico; transformación.

\section{ABSTRACT}

The confinement that originated in order to mitigate the COVID-19 pandemic has repercussive effects on different activities of daily life. This research seeks to identify transformations generated in the households of Ciudad Juarez, Chihuahua, through modifications in the use and order of essential areas. The methodology used is that of a mixed type, applying qualitative and quantitative tools, which account for the use of spaces and their transformations during the confinement by COVID-19. The results observed are identified changes that generate asynchronous spaces in the family's daily environment, modifying the previous organization and structure of the use of the dwelling.

Key words: synchronous; asynchronous; domestic space; transformation.

\section{INTRODUCCIÓN}

La rigidez que puede ocasionar cada habitación en la organización cotidiana de un hogar, se deriva de las rutinas estandarizadas que prevalecen en las actividades de las familias. En este caso, se reflexiona cómo las tendencias en la tecnología no han podido prever el cambio, debido a la emergencia que ha provocado la pandemia de la COVID-19, a pesar de encontrarnos en un mundo cada vez más digitalizado. ¿Cómo podríamos reconsiderar lo que significa un hogar y adaptar sus diseños a los comportamientos recién aprendidos? ¿Qué pasa si la raíz de la vivienda proviene de cómo vemos y utilizamos una casa en sus elementos fundamentales? (Overstreet, 2021). 
El sentido o concepto del espacio asincrónico en esta investigación hace referencia a los cambios que se han introducido en la vivienda y el modo de habitar, debido a la pandemia. Es decir, el espacio que debe corresponder a las nuevas demandas de la vida familiar, donde escuela, trabajo y hogar se han fusionado y entrelazado con actividades en tiempo y lugar.

El espacio sincrónico significa que la actividad debe realizarse al mismo tiempo y tales eventos a veces se llaman en tiempo real. Esto se debe a que las actividades son continuas y resuelven una necesidad compartida a su tiempo, pero no en un mismo momento.

Se plantea la aproximación al concepto de arquitectura flexible y dinámica, que en el hogar pasaron de ser espacios sincrónicos, reflejo de una vida cotidiana cambiante por la situación que obliga la contingencia derivada por la COVID-19.

La presente investigación se lleva a cabo en Ciudad Juárez, que se localiza al norte del estado de Chihuahua en México, frontera con la ciudad de El Paso, Texas, en Estados Unidos. Delimita al norte con el río Bravo; al poniente, con una sierra montañosa; y al sur, con una extensión desértica de arenales. Sus coordenadas geográficas son $31^{\circ} 44^{\prime}$ latitud norte, $106^{\circ} 05^{\prime}$ longitud oeste y 1835 msnm (Herrera, 2017: 21), teniendo una población de 1501551 habitantes (Inegi, 2020).

La obligación de permanecer en el hogar como disposición regulada por el gobierno federal con el interés de contrarrestar los efectos de contagio del virus SARS-CoV-2 y contrarrestar la pandemia de la COVID-19, ha provocado que los espacios domésticos en casa requieran otros enfoques que modifiquen los actuales prototipos de vivienda que se han repetido a lo largo del mundo. El encierro obligatorio conlleva a nuevas prácticas del cuidado y conservación de las casas, por lo que es urgente arreglarlas o modificar los espacios, mantener en estas relaciones interpersonales, contar con contactos estrechos y empáticos con las distintas generaciones, y en esto se incluyen muchas actividades realizadas por aburrimiento y que también dan paso a la imaginación. 
La arquitectura se considera generalmente como una disciplina de resolución de problemas, pero en la formación de los profesionistas de esta área se traduce en gran medida en la búsqueda de la estética. Las críticas que han emergido respecto a las soluciones arquitectónicas y de diseño están culturalmente a escala y formalmente fuera de contacto con el entorno actual (Monteys y Fuertes, 2001: 1). Al buscar solo la abstracción en la disciplina, se permanece alejado del objetivo de encontrar soluciones reales y prácticas de una arquitectura flexible, es decir, la propuesta de espacios multifuncionales y versátiles que correspondan a las distintas etapas de la vida del ser humano.

Se parte de nuevos requerimientos que son determinados por necesidades de transición que llevan a las actividades en línea o virtuales; estos requisitos arquitectónicos transitan de un modelo menos funcional a uno con mayor flexibilidad, que difieren de otras actividades realizadas en el hogar o en los distintos espacios domésticos previamente, condición que dificulta que los organismos profesionales puedan adecuarse en tiempo, ya que se requieren horas específicas de consulta y de actualización del conocimiento.

El resultado directo de estos cambios es que se han agravado los problemas sociales: violencia de género, falta o mala comunicación entre pares, abuso de poder, abuso verbal, dolor, miedo y ansiedad ante el desamparo e incertidumbre que esto genera; es el incremento de ambientes ${ }^{3}$ con mayor vulnerabilidad en donde el hogar no ha quedado exento, más bien es el epicentro del problema. Hemos comenzado a desarrollar la capacidad de descubrirnos a nosotros mismos y a los demás, a través de la autoobservación que mejora la conciencia, la creatividad y la capacidad práctica de las personas para las actividades de ocio (Holahan, 2012: 1).

Ante los panoramas sociales y políticos emergentes derivados de la pandemia, los espacios domésticos no cumplen con privilegiar las múltiples actividades que sus residentes deben incluir en

3 En esta investigación, el término ambiente (built environment, en inglés) se refiere a los espacios modificados por el ser humano que proporcionan el escenario para sus actividades diarias que van en escala, desde edificios y parques o áreas verdes hasta vecindarios, ciudades y el propio hogar (Grafova, 2008). 
el ámbito del hogar. Se dificulta la comunicación, la falta de conectividad, se incorporan otras actividades que como seres humanos o familias son múltiples con comportamientos, sentimientos y pensamientos que cambian constantemente. Aunque esto suele ser un problema arquitectónico, en estos tiempos se han expuesto las deficiencias de los edificios domésticos que no pueden satisfacer las necesidades humanas.

El objetivo de la arquitectura doméstica es convertirse en la protagonista del modelo emergente de aplicar la práctica profesional de los arquitectos, ya sea a nivel internacional, nacional y local, tomando en cuenta los materiales regionales y estando adaptada a las condicionantes climáticas y del lugar. Esto es importante para que cambie la forma en que los seres humanos sienten, piensan y se comportan. El espacio familiar debe ser una extensión del cuerpo y asegurar la vida feliz de las personas. La casa es nuestra capa exterior, "segunda piel”, alma y conexión con los demás.

La pandemia, que ha prevalecido durante la mayor parte de 2020, nos ha hecho preguntarnos acerca de nuestro estilo de vida. Muchos de nosotros nos hemos dado cuenta de que el espacio que alguna vez se consideró suficiente para las necesidades diarias, previo a la pandemia, ahora parece no funcionar de manera conveniente, al contar con otras actividades que no se realizan permanentemente en el hogar: educación y trabajo, especialmente cuando vivimos con otras personas.

La nueva realidad que impone la pandemia nos obliga a ser conscientes de la importancia de contar con múltiples y diferenciados espacios, desde la vivienda unifamiliar hasta la compartida en entornos rurales y urbanos. El entorno construido y la forma en que las personas interactúan en él, se fueron modificando inmediatamente, de manera inesperada y delimitada; por ello, las personas se volvieron conscientes de sí mismas en relación con el $\operatorname{lugar}^{4}$ que habitan.

4 El concepto de lugar en esta investigación hace alusión al propuesto por Frías (2003), en donde el ser humano necesita, a su vez, una morada donde pueda cobijar su alma y cuerpo; protegerse de las inclemencias del tiempo; dónde ofrecer un sosiego al sueño, un paraje al tiempo, que, a su vez, lo conduzca a otras formas de acción. Lugar donde volver habitualmente: dónde habitar, dónde guardar lo recolectado y guardarse 
La arquitectura tiene que mediar entre las necesidades percibidas del momento y aquellas que son desconocidas para el futuro; referente a necesidades inmediatas de nuestros cuerpos y el deseo de crear algo que durará más allá de las generaciones presentes (Tingley, 2020).

\subsection{Espacios asincrónicos}

De Certeau, Giard, \& Mayol (1994) plantean que habitar un lugar equivale a sentirse protegido, donde se separa la presión del cuerpo social sobre el individual; ahí plural de los estímulos, se filtra o, en todo caso, idealmente debería filtrarse. Las actividades cotidianas llevadas a cabo en los espacios domésticos que soportan las necesidades de descanso, alimento, recreación y aprendizaje son la suma de ambientes que se transforman en relación con la función que les corresponde. El baño, estancia o recámara son espacios sincrónicos y se han convertido en asincrónicos. La importancia de lo cotidiano se sitúa con lo que plantean De Certeau et al.:

Lo cotidiano es lo que se nos da cada día (o nos toca en suerte), lo que nos preocupa cada día, y hasta nos oprime, pues hay una opresión del presente. Cada mañana, lo que retomamos para llevar a cuestas, al despertar, es el peso de la vida, la dificultad de vivir, o de vivir en tal o cual condición, con tal fatiga o tal deseo (1994: 1).

Esta cotidianidad se ha establecido mediante rutinas y tiempos de uso, que permanecen en los horarios de escuela y trabajo con menos presencia de personas. Luego se identifica que:

Lo cotidiano nos relaciona íntimamente con el interior. Se trata de una historia a medio camino de nosotros mismos, casi hacia atrás, en ocasiones velada; uno no debe olvidar ese "mundo memoria", según la expresión de Piguy. Semejante mundo nos interesa mucho, memoria olfativa, memoria de los lugares de infancia, memoria del

a sí mismo. Lugar como centro y trasfondo donde discurre la vida, dónde ser (Frías, 2003). 
cuerpo, de los gestos de la infancia, de los placeres. Tal vez no sea inútil reiterar la importancia del dominio de esta historia "irracional", o de esta "no historia", como todavía la llama A. Dupront. Lo que interesa de la historia de lo cotidiano es lo invisible... (De Certeau et al., 1994: 1).

El trabajo en casa, las reuniones y la escuela virtual forman parte de una nueva normalidad a nivel mundial. Las medidas implementadas ante el avance de la COVID-19 alteraron el desarrollo de los vínculos familiares y laborales, así como la cotidianidad de miles de familias.

Dentro de los aspectos de diseño a considerar en esta nueva normalidad, se incluyen principios de planificación en donde se define un nuevo tipo de espacio entre multifacético, atractivo y productivo ante la inminente necesidad de llevar a cabo distintas actividades en el mismo momento.

Elementos como la proximidad entre las personas (personapersona, persona-tecnología, persona-necesidades) en espacios domésticos y cotidianos deben establecerse dentro de los principios de planificación, así como tomar en cuenta los nuevos hábitos de distanciamiento. Aunado a esto, la privacidad (visual, territorial, informativa y acústica) contribuye a reivindicar este tipo de espacios, creando o abriendo límites determinados por los usuarios. La personalidad (del espacio) conecta con la identidad de los usuarios, dando un nuevo enfoque que puede determinarse como espacio compartido o no.

Las posibilidades de colaboración, socialización, centralización y trabajo en casa ocurren en espacios, tanto domésticos (privados y semiprivados) como establecidos predeterminadamente para adaptarse a la nueva normalidad, pero los desafíos a los que se enfrenta el diseño de la arquitectura para hacer que estos dos tipos de espacios sean más convenientes son muy diferentes, lo que replantea la necesidad de creación de espacios asincrónicos.

Otro aspecto de la situación que involucra a la arquitectura actual en razón del confinamiento propiciado por la pandemia, es esta transformación de los mismos espacios, partiendo de que 
han sido identificados por los análisis conductuales que influyen o no en la posibilidad de lograr que los individuos y las familias se expandan; más bien se logran aprisionar y obstruyen las emociones y pensamientos, dando origen a la emergencia de espacios flexibles.

Los nuevos paradigmas del diseño definen premisas conceptuales diferentes, derivadas en principio por las medidas de confinamiento por la pandemia, y también enfocan los esfuerzos en la sostenibilidad económica, social y ambiental. Cuando la crisis se convirtió en una pandemia mundial nuestras prioridades en los espacios domésticos cambiaron inmediatamente.

La premisa conceptual se centra en la generación de espacios sincrónicos y asincrónicos. Lo sincrónico, según Terry y Linden (2020) significa que todos los involucrados que realizan una actividad deben efectuarla al mismo tiempo; tales eventos a veces se llaman en tiempo real o en vivo. Esto se debe a que las actividades son continuas y corresponden a una necesidad compartida o común del espacio. Por otro lado, lo asincrónico son aquellas actividades que llevan a cabo los participantes que pueden experimentar en eventos con distintos tiempos y usos diversos que tienen requerimientos de ambientes diferenciados, pero que están realizándose al mismo tiempo dentro del mismo espacio.

$\mathrm{El}$ espacio flexible fácilmente lo imaginamos con paneles que se mueven, muebles que se pliegan o cortinas que se despliegan. Estos espacios permiten una mayor diversidad en las funciones que pueden albergar otros elementos no movibles. Un espacio es flexible al adaptarse a distintas necesidades a lo largo de su vida útil. Esto se puede entender como una modificación continua del espacio, efectuada por los usuarios, o por una reutilización de una estructura, para convertirla en otro ambiente completamente distinto (Forqués, 2016).

Otra forma de entender la flexibilidad del espacio es por medio de su adaptabilidad arquitectónica, que se concibe como una cualidad espacial que ofrece dinamismo en la distribución interior con carácter dinámico de cambio y que responde a las sociedades y culturas que la generan, demandan y transforman; una 
arquitectura que les permita adaptarla a sus gustos y necesidades (Forqués, 2016).

La imposibilidad de acotar los modos de accionar contemporáneos en recintos determinados, sumada a los nuevos requerimientos de adaptabilidad y mutación constante de los microespacios, ha dado lugar a la manifestación de diversas estrategias arquitectónicas en pro de una búsqueda de la flexibilidad en el habitar (Maiztegui, 2019).

La flexibilidad no implica simplemente la necesidad de un cambio infinito y una quiebra de la fórmula aceptada hasta ahora. Por el contrario, se podría entonces definir la flexibilidad del espacio arquitectónico como la idea de espacio delimitado hacia el exterior y con diferentes posibilidades de distribución interior; como una cualidad del espacio construido para ser modificado cuando el uso así lo requiera, acercándose cada vez más a la idea de plantas libres con estructuras generalmente puntuales, núcleos fijos de servicio (baños, cocina, escaleras) y múltiples maneras de distribuir las áreas de permanencia (Forqués, 2016: 1).

En el contexto de la flexibilidad surge el loft, por ejemplo, cuyo origen se encuentra en la ciudad de Nueva York en el año de 1950, principalmente en tres de sus barrios: TriBeCa, SoHo y el Barrio Oeste. Estas zonas fueron las grandes protagonistas de una industria floreciente y, más tarde, olvidada. La necesidad de grandes espacios y las rentas elevadas de pisos y apartamentos hizo que las fábricas y almacenes en desuso cobraran un gran protagonismo para ciertos sectores de la población. En un principio los utilizaron estudiantes y artistas de una forma más o menos clandestina, pero con el tiempo fueron rehabilitados y adaptados a restaurantes, tiendas, estudios de pintura o fotográficos y galerías de arte. Rápidamente su uso se trasladó al ámbito doméstico, convirtiéndose en viviendas de lujo, espaciosas y confortables que invitan a la calma. En la actualidad, las viviendas tipo loft se han convertido en todo un fenómeno social que se traduce en una forma de vida vanguardista y de alto nivel de versatilidad en cuanto al uso de sus espacios (Zukin, 1989: 8). 
La percepción de la arquitectura es una secuencia de impresiones visuales, táctiles y sonoras, que se reciben con un ritmo análogo, que se emplea como lenguaje, y que los tiempos de la percepción, del número de imágenes, de la velocidad de esa aprehensión, etcétera, están regidos por leyes análogas que se emplean para construir el lenguaje, los pensamientos, las expresiones orales y, por lo tanto, la literatura, que es, a su vez, una destilación de la experiencia de la vida (Perucho, 2004).

Al contrario de los espacios asincrónicos, los sincrónicos, referidos a aquellos en los que se presenta un uso limitado e inflexibilidad a los paradigmas emergentes de la arquitectura, son resultado de una arquitectura rígida, inalterable, poco adaptable y poco ajustable.

Evaluando los paradigmas arquitectónicos tradicionales, se pone en evidencia que vivimos en edificios rígidos, inmóviles, inflexibles, que no aceptan cambios importantes, como si el ser humano y la naturaleza no registraran cambios durante su vida. Con excepciones, en las que es posible cambiar con efectos decorativos o ambientales algunos elementos del edificio (Hurtado, \& Sánchez, 2015).

Si escribir es la forma de realizar ideas, entonces la arquitectura es la forma de definir el espacio. Le Corbusier lo define en el área delimitada por las paredes de la casa como una extensión del cuerpo del individuo, su cuerpo mejorado y su estructura. Es el confidente silencioso y respetuoso de todo lo que sucede en el interior, un misterio visto desde el exterior. Estas apariciones son las que llevaron a la transformación de las casas de refugios a viviendas protegidas y la evolución de sus pieles a espacios más abiertos, convirtiéndolas en los rostros visibles de los conjuntos internos de sus residentes. A partir de entonces, la casa comenzó a agregar valor para realzar su función protectora original (Forqués, 2016: 1).

Todos estos conceptos no resuelven los requerimientos del paradigma generado por la pandemia; por ello, el espacio asincrónico es, sin duda, el tema a identificar en condiciones de coyuntura en la arquitectura. Es esta concepción del espacio que podrá, entonces, 
adaptarse a la nueva normalidad en situaciones emergentes y que se logra por la definición de ambientes múltiples con usos entrelazados diferentes en tiempo y actividad (Peña, \& Herrera, 2020).

En este trabajo se parte, entonces, del supuesto de que las viviendas en este momento de la pandemia no responden a los requerimientos que la coyuntura exige a las familias en su dinámica cotidiana y que la mayoría de los espacios sincrónicos, se tienen que adaptar a usos asincrónicos.

\subsection{Metodología}

Esta investigación se desarrolla a partir de una metodología de tipo mixto, ya que se utilizan herramientas del método cuantitativo (gráficas, registros e indagación documental) y del método cualitativo (opinión y percepción) de acercamiento con algunos actores clave. Para lograrlo, se consideran los siguientes pasos:

En la elaboración de la introducción se identifican los conceptos más relevantes del nuevo paradigma del diseño mediante investigación documental de distintos autores obtenidos de textos o internet, que aportan a la profundización del tema.

En la construcción de la discusión teórica sobre el espacio flexible, sincrónico o asincrónico, se desarrolla a manera de ensayo la reflexión de los aportes de distintos autores para la construcción propia del concepto de espacio sincrónico y asincrónico.

En la identificación de las transformaciones generadas en el ámbito de los hogares, se registran las distintas modificaciones en uso y espacio de áreas esenciales para la vida cotidiana en la vivienda mediante la revisión de respuestas de la encuesta aplicada y el análisis de ambientes típicos en el interior de algunas viviendas.

En el análisis de los resultados, se utiliza la triangulación de la información de percepción y cuantitativa, aportando a la reflexión sobre los espacios sincrónicos que han sido modificados y que tienen uso asincrónico. La comparativa entre resultados permite la identificación de constantes.

En la elaboración de las conclusiones, se describen los hallazgos más relevantes de la investigación, considerando los elemen- 
tos que den cuenta de las tendencias que están transformando la dinámica de los espacios domésticos pos-COVID-19.

En la validación de la muestra de la encuesta, se utilizaron procedimientos distintos en la aplicación derivados de la coyuntura de la pandemia. De esta manera, se recurrió a la difusión amplia de la encuesta por medios digitales, debido a la limitante de sana distancia determinada por el Sector Salud como requisito indispensable para disminuir la transmisión del contagio. Se realizó una encuesta con un total de 38 preguntas, a través del software Google Forms, que permite recolectar los resultados en Excel y, posteriormente, ser organizados en una plataforma estadística de spss versión 19. El total de cuestionarios aplicados fueron 351 en Ciudad Juárez, aportando de manera general información de una visión panorámica de la problemática sobre el tema.

Esta encuesta se envió por WhatsApp en formato del software forms y, posteriormente, se utilizó el programa Excel para transferir los datos a la plataforma spss versión 19. Se integran los resultados de tipo perceptual, que corresponde a la opinión de los usuarios de espacios domésticos en general, para conocer la experiencia que han tenido dentro de la vivienda durante este confinamiento y ayudar a comprender las áreas de oportunidad que se percibieron. Esta información aporta a la reflexión respecto al debate de los paradigmas emergentes en la arquitectura posCOVID-19.

La encuesta fue respondida por mujeres (64\%) y hombres (34 \%) que residen en Ciudad Juárez, Chihuahua, en donde se puede considerar que se obtuvo una muestra de la visión acerca de las transformaciones surgidas a través del confinamiento por la pandemia de la COVID-19. La característica de la muestra es que cumple con los estándares estadísticos de $95 \%$ de validez y $5 \%$ de posible error. 


\section{RESULTADOS}

Los resultados aportan a la reflexión de la problemática que prevalece en el diseño de la vivienda, al plantear espacios sincrónicos con usos limitados en estos momentos de la pandemia.

En cuanto a los espacios asincrónicos es posible identificar aquellas áreas que han sido modificadas o convertidas en esta modalidad, debido a la emergencia de adaptarla para la enseñanza y el trabajo. En este sentido, se observa el mayor número de áreas asincrónicas en espacios domésticos de las viviendas, evidenciando que este mejoramiento es parte del paradigma emergente de diseño en la arquitectura de México y el mundo.

\subsection{Espacios asincrónicos y sincrónicos}

En la discusión teórica, los espacios sincrónicos son los que fueron diseñados para un uso determinado; en cambio, los espacios asincrónicos se emplean con actividades entrelazadas en tiempo y actividad, dificultando la convivencia y ambiente de tranquilidad en el hogar. Estos espacios sincrónicos se han modificado en 59 $\%$ de los hogares de las personas que resolvieron el cuestionario.

Uno de los elementos de importancia durante la pandemia, y que se vuelve un tema a resaltar, es el de paradigmas emergentes, que hace referencia al número de integrantes que habitan en una vivienda, siendo de la siguiente manera:

a) El $2 \%$ es ocupada por 1 persona

b) En el $22 \%$ de los casos es habitada por 2 personas

c) El $19 \%$ de las respuestas corresponde a casas con 3 integrantes

d) El $31 \%$ es ocupada por 4 personas

e) En el $18 \%$ de los casos se integra por 5 miembros

f) El $7 \%$ es ocupada por 6 personas y en el $1 \%$, por 7 habitantes 
En este caso las familias que tienen más de cuatro integrantes, se han visto conflictuadas en el uso de espacios sincrónicos y, por ello, 59 \% ha modificado esas áreas (véase figura 1).

Figura 1. Ocupantes por vivienda

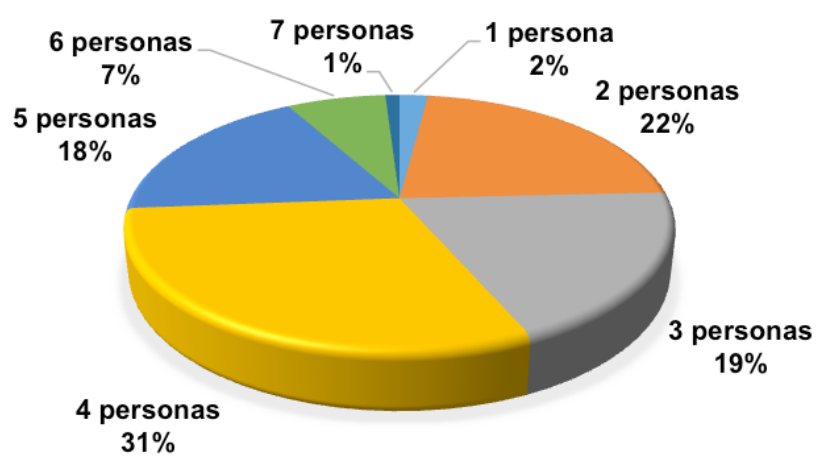

Fuente: elaboración propia con base en información de 351 encuestas de opinión aplicadas virtualmente.

Se puede observar que los espacios sincrónicos, que son áreas creadas con una finalidad y característica específica e inflexibles a cambios y transformaciones, han tenido que adaptarse por la contingencia sanitaria, convirtiéndose en espacios asincrónicos que funcionan de manera diversa, dando respuesta a eventos desfasados o no en el tiempo y actividad; de esta manera, la sala o la recámara se han convertido en sitios que se adaptan a situaciones emergentes con distintas actividades y que demandan usos incompatibles, incluso.

Uno de los elementos de importancia durante la pandemia, y que se vuelve un tema interesante a resaltar respecto a los paradigmas emergentes en el diseño de la arquitectura pos-COVID-19, es el distanciamiento social, ya que a la mayoría de los espacios que cumplen el mínimo recomendado por el Reglamento de Construcción es casi imposible hacerles una conversión, debido a lo limitado del espacio. 
Mencionan Peña y Herrera (2020) que esto afecta la movilidad en el hogar:

Esto nos lleva a reflexionar que la actividad cotidiana ha sido irrumpida con la permanencia de la familia en casa, que durante dos meses no fueron al trabajo, se realizaron actividades escolares con los hijos y se tenían que resolver las exigencias de atención del hogar (32).

Las condiciones del distanciamiento social han implicado mantener actividades académicas o transmitir clases en línea, el cierre de negocios, trabajar desde casa, permanecer en aislamiento social o en cuarentena las veinticuatro horas, interrumpir reuniones masivas (conferencias, conciertos), evitar el acercamiento con seres queridos y solo tener comunicación a través de dispositivos electrónicos (Gómez, 2020: 3), lo que nos adentra en la reflexión ante los paradigmas emergentes del diseño en la arquitectura y la concepción de espacios pensados como asincrónicos, que permitan una mayor movilidad y mantener las funciones con una ocupación conveniente.

En ese sentido, la distancia al interior de la vivienda mantiene a las familias o personas en situaciones poco convenientes para cumplir con esta disposición, ya que el $15 \%$ de las personas encuestadas mantiene menos de $1 \mathrm{~m}$ de separación; el 20 \%, $1 \mathrm{~m}$ de alcance; el 30 \%, 1.50 m de distancia; y el $34 \%$ declara que desconoce la separación entre ellos en el interior de su vivienda, lo cual demuestra que más del $90 \%$ de los encuestados no puede establecer una distancia conveniente en esta situación de emergencia generada por la pandemia (véase figura 2). 
Figura 2. Distancia al interior de la vivienda que mantiene con su familia o personas que la habitan

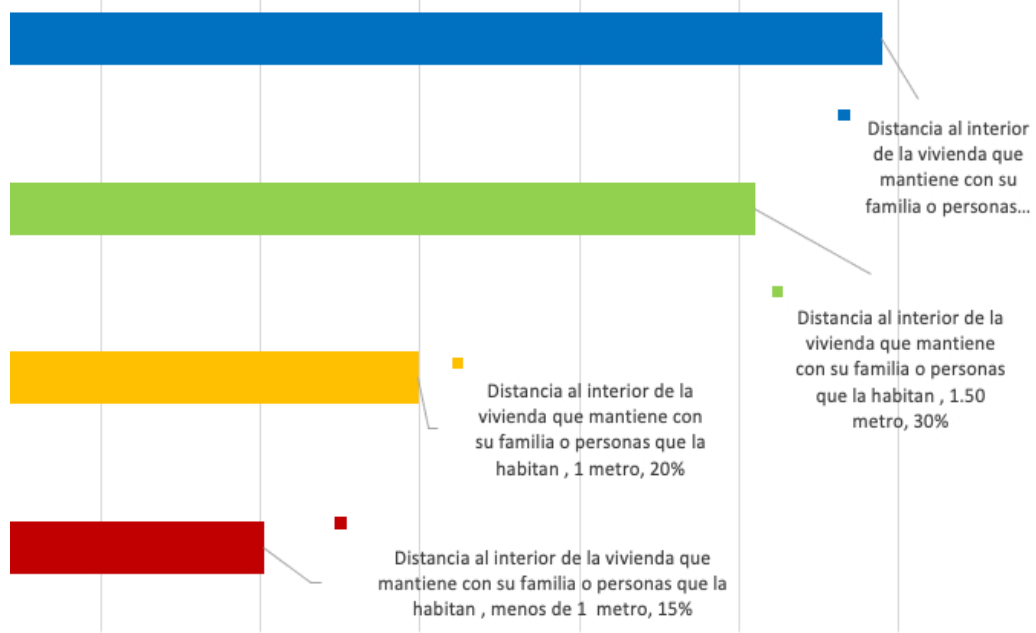

Fuente: elaboración propia con base en información de encuestas de opinión.

Ante esta situación existen intersecciones dentro de los espacios domésticos sincrónicos, que podrían inhibir el distanciamiento social como medida de prevención ante la pandemia, al mostrar que $35 \%$ de las personas encuestadas mantienen $1 \mathrm{~m}$ o menos de distanciamiento social reglamentado en México, lo que podría resolverse con los espacios asincrónicos que permitan la diversidad de actividades en las viviendas generando una mayor amplitud.

En esta investigación se observa que en los resultados de la distancia al interior de las casas, se incorpora la percepción de riesgo que se expresa ante la pandemia, ya que $54 \%$ de los encuestados menciona estar preocupado por contagiarse y $20 \%$ está muy preocupado, en contraparte de quienes perciben un menor riesgo, ya que $21 \%$ se siente tranquilo, $4 \%$ se siente seguro y 1 $\%$ se siente muy tranquilo estando en su vivienda durante esta contingencia de salud (véase figura 3). 
Figura 3. Percepción de riesgo ante la pandemia de la COVID-19

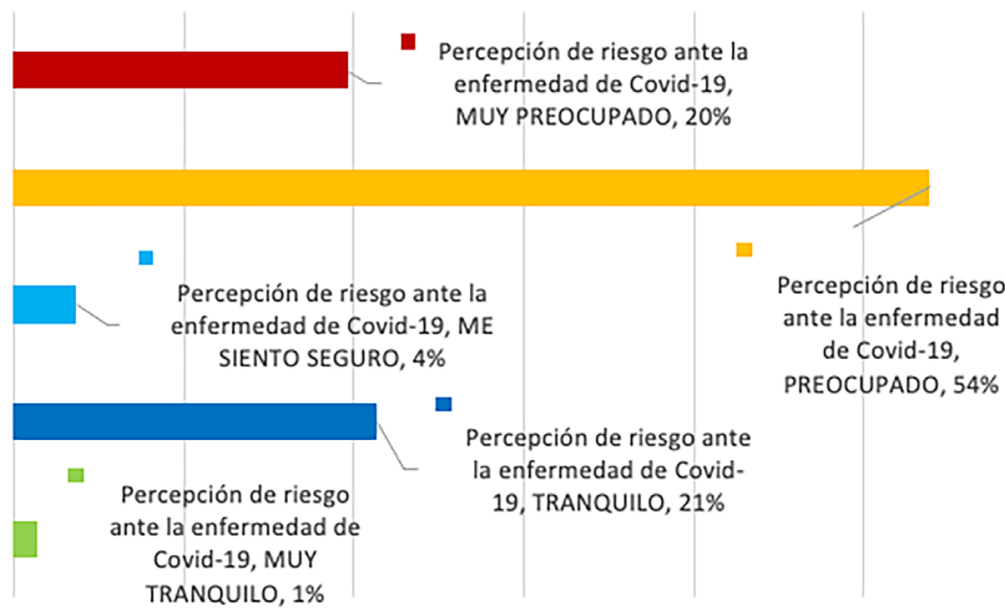

Fuente: elaboración propia con base en información de encuestas de opinión.

En la percepción de riesgo, se considera que existe relación con los factores espaciales, donde con base en las respuestas que se muestran a continuación y el traslado de actividades esenciales a los espacios domésticos transformados en asincrónicos por la emergencia, podría ser un condicionante clave respecto a la relación e interpretación del entorno inmediato.

En relación con los espacios utilizados con más frecuencia o en los que se permanece por más tiempo durante el confinamiento, en donde comienza a tomar forma la relación entre los espacios sincrónicos transformados en asincrónicos, $47 \%$ de las personas encuestadas respondió que pasa más tiempo en la sala-comedor; $19 \%$, en la sala de estudio; $12 \%$, en la cocina; $2 \%$, en el patio o jardín; y $20 \%$, en otro espacio (taller, patio, balcón, etcétera) (véase figura 4).

Los escenarios a los que nos enfrentamos están provocando una readaptación a todos los niveles en donde las soluciones arquitectónicas se vuelven trascendentales en la manera en que se va afrontar la nueva normalidad que nos espera (pos-COVID-19. La arquitectura se encuentra en un momento clave en el que hay que 
anticiparse y tener conciencia acerca de la importancia de saber adaptar los ambientes a cualquier situación.

\subsection{Espacios sincrónicos vs asincrónicos}

Al describir los espacios, como la sala, a través de su entendimiento como espacio sincrónico, se considera que se utiliza para ver la televisión, convivir, recibir visitas, mas si funciona como espacio asincrónico tiene estos usos, pero también al mismo tiempo se realizan actividades de trabajo, educación y de esparcimiento de manera virtual.

Figura 4. Espacios utilizados con más frecuencia o en los que se permanece más tiempo durante la pandemia de la COVID-19

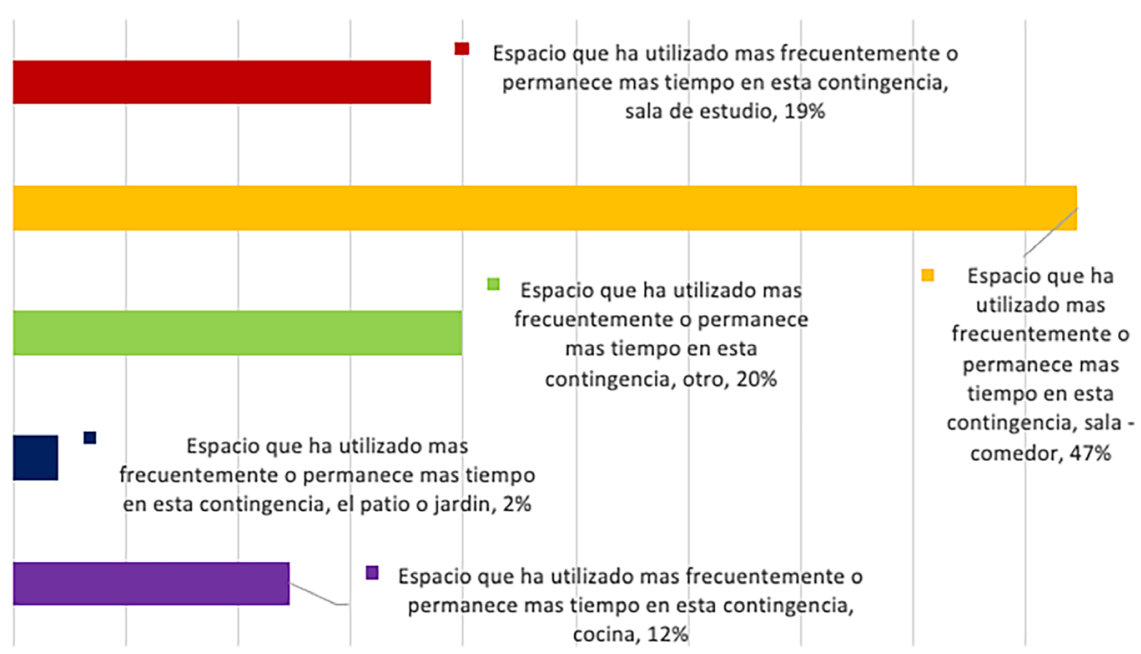

Fuente: elaboración propia con base en información de encuestas de opinión.

Respecto a la discusión principal de esta investigación, se presentan un mayor número de áreas asincrónicas en espacios domésticos de las viviendas, transformándose de áreas sincrónicas, como dormitorios, salas, cocinas, patios/jardines, comedores u otros en espacios que se utilizan de forma frecuente para realizar trabajo, educación o esparcimiento. Las personas encuestadas 
comparten estos cambios de la siguiente manera: $38 \%$ convirtió la recámara; $30 \%$, el comedor; $17 \%$, el cuarto de estudio; $4 \%$, la cocina; $9 \%$, otro espacio (talleres, patios, balcones, etcétera), mientras que $3 \%$ no aplica, porque no realiza esas actividades de forma virtual (véase figura 5).

Figura 5. Espacio que se usa de forma frecuente para realizar el trabajo, educación o esparcimiento en la casa

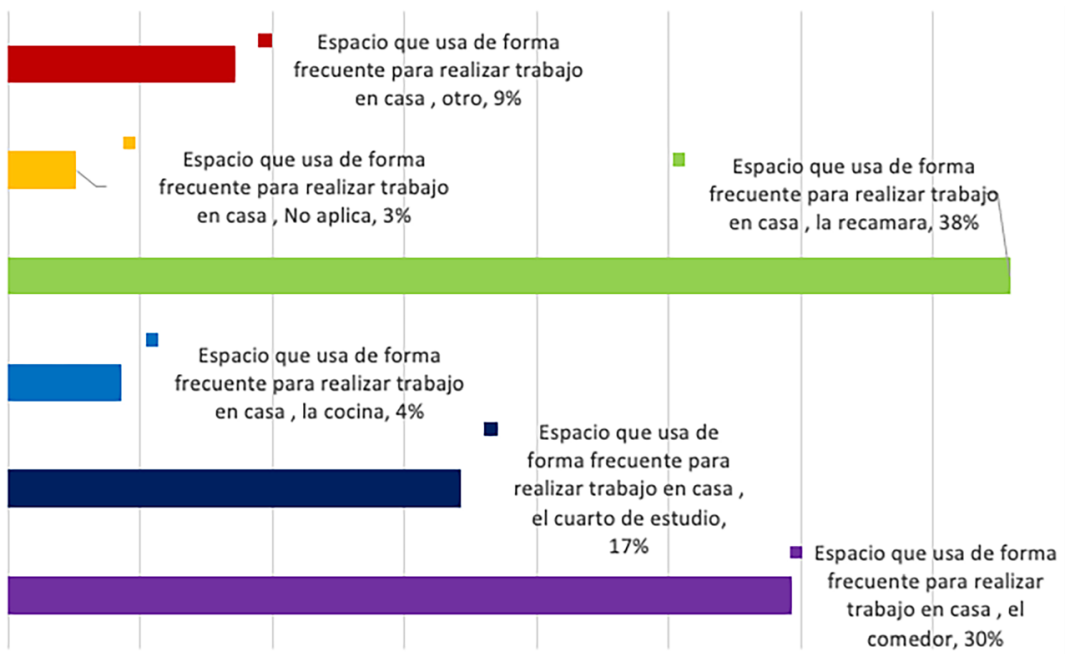

Fuente: elaboración propia con base en información de encuestas de opinión.

Un atributo relevante en la era pos-COVID-19 es la emergencia de repensar los espacios con múltiples requerimientos para permitir el desempeño de las distintas actividades, como el trabajo, la educación y otras que tuvieron que trasladarse a los hogares debido al confinamiento; en donde hay que maximizar la luz natural y el aprovechamiento de la ventilación cruzada, amortiguar el ruido o las conversaciones con la posibilidad de cambiar la distribución interior y el aislamiento, que se derivan en paradigmas extraordinarios por abordar.

En cuanto a la necesidad de reordenar algún espacio para adaptarlo a otra función, 59 \% lo llevó a efecto y 41 \% no lo hizo, por lo que es una situación cuya emergencia alcanza a un grupo 
de población numeroso (207 hogares). Debido a esto, las viviendas y los espacios domésticos deben ganar en versatilidad en donde la nueva normalidad instaura exigencias de espacios asincrónicos con diversidad y dinamismo, que cobran especial importancia frente a la rigidez de los espacios sincrónicos (funcionales) actuales (véase figura 6).

Figura 6. Necesidad de reordenar un espacio para adaptarlo a otra función

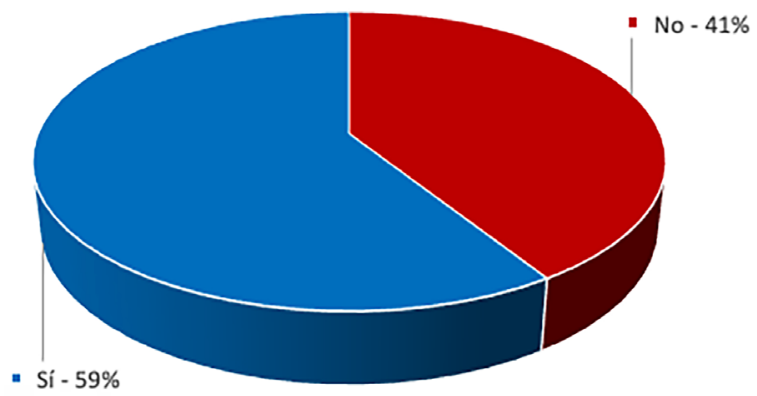

Fuente: elaboración propia con base en información de encuestas de opinión.

La vivienda se ha convertido en defensa de primera línea contra el coronavirus. Nunca había sido el espacio en donde se dirime la condición de vida o muerte (Hábitat México, 2020). El ambiente estresante de la estadía en el hogar, especialmente en viviendas pequeñas y saturadas en las que se puede encontrar una solución viable, definiendo espacios asincrónicos como paradigma emergente pos-COVID-19.

\section{ANÁLISIS DE RESULTADOS}

La pandemia ocasionada por la COVID-19 nos ha permitido revalorar que los espacios domésticos no solo son en el interior de las viviendas, sino que se trasladan al exterior en una búsqueda 
de aire libre y naturalidad. En este sentido, la actividad cotidiana presenta la emergencia de uso de espacios que deben solucionar la multiplicidad de ocupaciones de lo común y también de lo emergente.

Por ejemplo, los patios, terrazas, balcones y jardines se transforman en su uso en cotidianos (pospandemia) cuando anteriormente se utilizaban en situaciones extraordinarias o eventuales. Zonas exteriores que, a su vez, pueden integrarse con los espacios interiores dentro de la vivienda. Estancias unidas a terrazas con la posibilidad de abrirse o cerrarse en función de las circunstancias (véase figura 4).

El nuevo paradigma de la arquitectura doméstica, se replantea a partir de la recámara, la cocina, el estudio y el espacio verde (patio, terraza-jardín, balcón, jardín vertical, cualquiera que el tamaño permita adaptar e incorporar a la vivienda), estancias que prodigan todo: trabajar, descansar, cultivar el cuerpo, el ocio y la vida lúdica, individual, familiar e, incluso, colectiva, y que pueden transformarse en el tiempo y ser usadas, incluso si hay pandemia (véase figura 5). Se revalúan algunas áreas que consumen espacio y que han ido perdiendo jerarquía y uso en los modos de vida actual, como la sala y el comedor formal, en las cuales posibilitar la flexibilidad, la transformación y la cohabitación de actividades debe ser una premisa central.

Los espacios presentan una reconfiguración del uso cotidiano en 59 \% de los que existían (véase figura 6). En este caso, el interior no es suficiente para atender las actividades diarias durante las 24 horas de uso permanente; al igual, el exterior ha tomado relevancia para ocupaciones permanentes.

La resignificación de estos espacios en donde los cambios de hábitos y las rutinas cumplen una función relevante en relación con su uso; los espacios asincrónicos en donde eventos con distintos tiempos y usos diversos que tienen requerimientos de ambientes diferenciados, pero que están realizándose al mismo tiempo. Por ejemplo, se contestan llamadas de trabajo mientras se cocina, se ayuda en las tareas de las hijas e hijos o se tienen 
clases o reuniones virtuales a la vez que la familia se encuentra en tiempo de convivencia.

Según la doctora Carolina González (2021) de la Universidad de La Frontera, en Chile, "Las casas en la actualidad parecen atender a una perspectiva sociófuga, que nos expulsa fuera del hogar para satisfacer necesidades; en lugar de proveernos de espacios versátiles, que se adapten a nosotros y no al revés". La emergencia ha puesto en evidencia estas necesidades en la vivienda en donde es inevitable que las nuevas formas de trabajo, de vida y de relación social, se incorporen a nuestras costumbres a partir de ahora.

La convivencialidad como valor ético intrínseco de una sociedad en la que la libertad individual, se realiza en interdependencia con otros y en relación con el entorno en que vivimos y donde nos importa cómo vive el otro.

\section{CONCLUSIONES}

No solo los parques públicos, sino también los balcones, patios y jardines que antes se empleaban como espacios exteriores, ahora ofrecen una oportunidad para respirar aire fresco y un tiempo donde se puede pasar lejos del interior. El nuevo tipo de espacio doméstico ante las condiciones de la emergencia sanitaria nos lleva a repensar en soluciones habitacionales con la creación de espacios asincrónicos y versátiles, que definan la creación de las nuevas viviendas.

Nuestro espacio privado es especialmente importante. Las limitaciones nos hicieron pensar en nuevas configuraciones para adaptarnos a un estilo de vida más acotado al ámbito de la familia y compartido con parientes y amigos cercanos. La calidad y comodidad de nuestras casas estará en las prioridades de una lista donde la emergencia resuelva la diversidad.

La trascendencia de concebir viviendas resilientes, de mayor superficie, higiénicas y que den paso a espacios asincrónicos, es una necesidad que se debe incorporar de manifiesto en los paradigmas emergentes en el diseño de la arquitectura pos-COVID-19; en donde se cultive el nuevo movimiento frente a la vida, que 
comprenda no solo las dimensiones físicas, sino también los significados sociales y afectivos del constructo de hogar.

Estos espacios asincrónicos ayudarán a lo que Illich (1978) llama "la reconstrucción de la convivencialidad"; valor que ha emergido durante el uso del espacio cotidiano en este momento de pandemia.

\section{BIBLIOGRAFÍA}

De Certeau, M., Giard, L., \& Mayol, P. (1994). La invención de lo cotidiano 2: inventar, cocinar (1.a ed.). Universidad Iberoamericana/ITESO.

Durón Morales, L. F., \& Peña Barrera, L. (2021). La formación del arquitecto en el debate de la sustentabilidad en la enseñanza de tres universidades. En: Investigación en arquitectura. Ciudades accesibles, sustentables y habitables (pp. 159-180).

Forqués Puigcerver, N. (2016). La flexibilidad en la arquitectura. Mito, Rev. Cult., 30.

Frías, L. G. (2013). Lugar y arquitectura. Reflexión de la esencia de la arquitectura a través de la noción de lugar. Arquitectura revista, 9(2), 161-169. https://doi.org/10.4013/arq.2013.92.09.

González, C. (2021). El espacio doméstico: el otro gran escenario del COVID-19, Entrevistado por Carrasco Salas, Pamela. Universidad de la Frontera.

Gómez-Calles, T. J. (2020). Intersección de pobreza y desigualdad frente al distanciamiento social durante la pandemia covid-19. Rev. Cub. Enf., 36, 1-15.

Grafova, I. (2008). Overweight Children: Assessing The Contribution Of The Built Environment. Prev Med., 47, 304.

Hábitat México (2020). Vivienda y covid-19. Hábitat para la humanidad. https://www.habitatmexico.org/article/vivienda-ycovid\#: :text=La vivienda es ahora, una cuestión de vida o muerte 
Herrera Sosa, L. C. (2017). Evaluación térmica del material isoblock en el clima cálido seco de Ciudad Juárez, México. Rev. Háb. Sust., 7(2), 18-27.

Holahan, C. (2012). Psicología ambiental: un enfoque general. Grupo Noriega Editores/Limusa.

Hurtado, M., \& Sánchez, A. (2015). Arquitecura dúctil. International Conference Arquitectonics Network: Architecture, Education and Society, 3-5.

Illich, I. (1978). La convivencialidad. Prefacio. Introducción.

Instituto Nacional de Estadística y Geografía (Inegi) (2020). Scince 2020.

Maiztegui, B. (2019). Ejemplos de espacios flexibles por medio de divisiones móviles en Argentina. Archdaily.mx https://www. archdaily.mx/mx/924843/ejemplos-de-espacios-flexibles-pormedio-de-divisiones-moviles-en-argentina

Monteys, X., \& Fuertes, P. (2001). La casa collage: un ensayo sobre la arquitectura de la casa. En: G. Gili (Ed.), Persepsi Masyarakat Terhadap Perawatan Ortodontik Yang Dilakukan Oleh Pihak Non Profesional (1.a ed., Vol. 53, Art. 9).

Overstreet, K. (2021). ¿Qué es realmente un hogar y cómo planificamos su futuro? Archdaily.mx https://www.archdaily.mx/ mx/954510/que-es-realmente-un-hogar-y-como-planificamossu-futuro

Peña, L., \& Herrera, L. (2020). La vivienda construida en serie y la influencia de la normatividad para las condiciones de habitabilidad en Ciudad Juárez, Chihuahua, México. Edähi, Bol. Cient. Cienc. Soc. Hum. Icshu, 9(17), 26-34.

Perucho Lizcano, J. (2004). La estructura inflexible de la obra arquitectónica (como reflejo del sentido del espacio en el mundo de las ideas y en paralelo con la estructura formal de la obra literaria).

Terry, L., \& Linden, S. (2020). How Do I Decide if my Course or Program Should Be Blended Online or Hybrid sp. Digital Teaching \& Learning-Laureate International Universities.

Tingley, K. (2020, 28 de junio). La arquitectura podría ayudar a adaptarnos a la pandemia. La propagación del virus no es solo una 
crisis de salud; también es un problema de diseño. The New York Times. https://www.nytimes.com/es/2020/06/28/magazine/ arquitectura-pandemia.html?fbclid=IwAR37U4uGRI55b9CRBZPBd-5Lz-AYFEXhpknszX2tv3EKKIn-FeoKDPI2Io

Zukin, S. (1989). Loft Living: Culture and Capital in Urban Change. Rutgers University Press. https://scholar.google.es/ scholar?hl=es\&as_sdt=0\%2C5\&q=loft+mexico\&btnG=\#d=gs_ cit\&u=\%2Fscholar\%3Fq\%3Dinfo\%3AxNZdIXjmpGAJ\%3Aschol ar.google.com\%2F\%26output\%3Dcite\%26scirp\%3D1\%26hl\%3D es

\section{AGRADECIMIENTOS}

Al Consejo Nacional de Ciencia y Tecnología (Conacyt) por el financiamiento de la investigación que aporta los datos estadísticos.

A la doctora María Eugenia Molar Orozco, de la Universidad Autónoma de Coahuila, coordinadora de esta investigación.

Al M. D. H. José Humberto Campuzano de la Torre, de la Universidad Autónoma de Ciudad Juárez (UACJ), por su asesoría en el uso del instrumento estadístico para la correlación de datos. A la institución académica que estamos adscritos (UACJ), que favorece estos vínculos de redes académicas entre los docentes. 